\title{
Influence of cardiac resynchronization therapy on oxidative stress markers in patients with chronic heart failure
}

Wojciech Krupa ${ }^{1}$, Małgorzata Rozwodowska ${ }^{2}$, Sławomir Sielski ${ }^{1}$, Ewa Czarnecka-Żaba ${ }^{3}$, Tomasz Fabiszak ${ }^{1}$, Gerard Drewa ${ }^{3}$, Michał Kasprzak ${ }^{1}$, Jacek Kubica ${ }^{1}$

${ }^{1}$ Department of Cardiology and Internal Medicine, Collegium Medicum, Nicolaus Copernicus University, Bydgoszcz, Poland

${ }^{2}$ Department of Geriatrics, Collegium Medicum, Nicolaus Copernicus University, Bydgoszcz, Poland ${ }^{3}$ Department of Medical Biology, Collegium Medicum, Nicolaus Copernicus University, Bydgoszcz, Poland

\begin{abstract}
Background: The aim of the study was to assess changes of substances of oxidative stress in patients treated with cardiac resynchronization therapy (CRT).

Methods: The study comprised 51 patients with median age of 66 years. The presence, severity, and changes of oxidative stress during CRT were assessed and expressed as malondialdehyde (MDA) concentration in plasma or in red blood cells. Antioxidant activity was assessed by the activity of catalase (CAT), superoxide dismutase (SOD) and glutathione peroxidase (GPX) in red blood cells. The concentration and activities were analyzed directly prior to implantation, 2-5 days after the procedure and after 6 months. Follow-up (6 months) included clinical, echocardiographic and implanted device assessments.
\end{abstract}

Results: During the follow-up, $14 \%$ patients died. The median percentage of biventricular pacing was $99.29 \%$. After 6 months $88 \%$ of patients improved NYHA, $12 \%$ remained as non-responders. Left ventricular ejection fraction increased from median $21.5 \%$ to $29 \%(p<0.05)$. Left ventricular end-diastolic diameter decreased significantly from a median of $69.5 \mathrm{~mm}$ to $63 \mathrm{~mm}(p<0.05)$. After a 6 -month study, MDA plasma concentration and the activity levels of each antioxidant enzymes (CAT, SOD, GPx) showed a statistically significant reduction $(p<0.05)$. Changes concerning MDA concentrations in red blood cells remained statistically insignificant.

Conclusions: Resynchronization effect oxidative stress by reducing plasma concentration of MDA, CAT, SOD and GPX. (Cardiol J 2014; 21, 5: 576-582)

Key words: heart failure, cardiac resynchronization, oxidative stress

\section{Introduction}

The prevalence of chronic heart failure (HF) in the general population amounts to $2-3 \%$ and rises with age up to $10-20 \%$ in elderly populations aged
$70-80$ years [1]. If associated with uncorrectable causes and irreversible myocardial damage, the prognosis of the disease is poor. The presence of HF symptoms increases the probability of further deterioration of left ventricular (LV) function

Address for correspondence: Wojciech Krupa, MD, PhD, Department of Cardiology and Internal Medicine, Collegium Medicum, Nicolaus Copernicus University, ul. Skłodowskiej-Curie 9, 85-094 Bydgoszcz, Poland, tel: +48 525854023 , fax. +4852 5854024, e-mail: wkrupa@cm.umk.pl

Received: 15.12.2013

Accepted: 04.07.2014 
and imposes a risk of life-threatening ventricular arrhythmias. The New York Heart Association (NYHA) reports a yearly mortality rate for patients with NYHA class III HF to be $20 \%$, with escalation up to $50 \%$ among class IV HF patients.

Mechanisms of death in HF patients are related to their severity. In the population of patients with mild or moderate HF left ventricular ejection fraction (LVEF) remains a strong predictor of prognosis of sudden cardiac death - in this population sudden cardiac death accounts for the majority of total mortality causes $[2,3]$.

Cardiac resynchronization therapy (CRT) is a well-recognized method for the treatment of $\mathrm{HF}$, in patients with refractory symptoms, despite optimal medical treatment and the presence of ventricular asynchrony [4].

Beneficial effects of CRT regarding improvement in clinical status, the quality of life and, most importantly, mortality reduction have been proven in large clinical trials [5-10].

However, approximately $20-30 \%$ of patients would not respond to this therapy [11-13].

The current European Society of Cardiology guidelines recommend CRT (class I indication) as a method for HF treatment in patients presenting with sinus rhythm, QRS complex duration $\geq 120 \mathrm{~ms}$, LVEF $\leq 35 \%$, and symptoms (NYHA class III-IV) despite optimal pharmacological treatment $[1,8]$. In those with life expectancy exceeding 1 year, implantation of a CRT defibrillator (CRT-D) is recommended [1, 14].

The impairment of LV function leads to activation of many neurohumoral mechanisms, crucial particularly for the prognosis and treatment options. The pivotal neurohumoral mechanisms include activation of the renin-angiotensin-aldosterone system and the catecholaminergic nervous system [15]. One of the mechanisms of less established pathophysiologic role in CHF in humans is oxidative stress in the failing heart [16].

Oxidative stress may lead to cardiac tissue damage due to imbalance between increased production of reactive oxygen species (ROS) and impairment of their neutralization by antioxidant defense systems [17]. ROS are constantly formed in the human body and removed by antioxidant defenses. An antioxidant is a substance which, when present at low concentrations compared to that of an oxidizable substrate, significantly delays or prevents oxidation of that substrate. Antioxidants can act by scavenging biologically important reactive oxygen species (e.g. $\left.\mathrm{O}_{2}^{-} \cdot \mathrm{H}_{2} \mathrm{O}_{2}, \cdot \mathrm{OH}\right)$ by preventing their formation, or by repairing the damage that they cause. One problem with scavenging-type antioxidants is that secondary radicals derived from them can often do biologic damage themselves.

Increased production of ROS observed in $\mathrm{HF}$ derives from several pathogenetic disturbances featuring catecholamine auto-oxidation, mitochondrial leak, recurrent ischemic episodes, and post reperfusion microcirculatory damage, as well as from the involvement of biologically active substances such as angiotensin II, xanthine oxidase, nitric oxide synthase and cytokines [16].

ROS contribute to HF etiopathogenesis by induction of apoptosis and myocyte necrosis [18], as well as by activating the matrix metalloproteinases and thus, promoting heart muscle fibrosis and harmful remodeling $[18,19]$.

Heart failure is characterized by increased ROS formation on the one hand, accompanied by a simultaneous drop in the activity of antioxidant enzymes on the other, with the latter resulting from growing consumption of antioxidant enzymes for neutralization of ROS [17].

Excessive ROS production in the failing heart is indirectly indicated by the presence of lipid and protein peroxidation products and could be measured in laboratory tests. One of the most popular products in laboratory practice is the thiobarbituric acid-reactive substances (TBARS). TBARS as important lipid peroxidation products, with their main component - malondialdehyde (MDA), are indicators of ROS [16, 20].

The aim of the study was to assess changes of plasma and erythrocyte MDA concentration and the activity of antioxidant enzymes (catalase [CAT], superoxide dismutase [SOD] and glutathione peroxidase $[\mathrm{GPx}]$ in red blood cells) in patients treated with CRT.

\section{Methods}

The study group comprised 51 patients aged 42-81 years (median age 66, ranging 59-72) admitted to The Department of Cardiology, Antoni Jurasz University Hospital of Collegium Medicum in Bydgoszcz, between November 2011 and December 2012 for CRT device implantation due to symptomatic HF, according to the criteria given above. For the final analysis 49 patients were included. Exclusion of 2 patients resulted from switching off the biventricular pacing due to permanent diaphragmatic nerve stimulation (1 patient) and lack of laboratory tests at 6 -month follow-up due to blood sample damage (1 patient). 
Table 1. Basic characteristics of patients with heart failure qualified for CRT.

\begin{tabular}{lc}
\hline Number of patients & 51 \\
Age [years] & 66.3 \\
Sex (male/female) & $40 / 11$ \\
Etiology of heart failure: & \\
Ischemic (no of patients) & $61 \%(\mathrm{n}=31)$ \\
Non-ischemic & $39 \%(\mathrm{n}=20)$ \\
NYHA classification: & 5 \\
II & 42 \\
III & 4 \\
IV & $21.5 \%$ \\
Echocardiography: & 69 \\
Ejection fraction [\%] & \\
LVEDD [mm] & 46 \\
Electrocardiography: & 4 \\
Sinus rhythm & 1 \\
DDD RV pacing & \\
Atrial fibrillation & $20.2(120-200)$ \\
QRS duration [ms] & \\
Comorbidities: & $33 \%(\mathrm{n}=17)$ \\
Diabetes mellitus & $45 \%(\mathrm{n}=23)$ \\
Hypertension &
\end{tabular}

CRT - cardiac resynchronization therapy; NYHA - New York Heart Association; LVEDD — left ventricular end-diastolic diameter

Demographic and medical characteristics of the patients are presented in Table 1 . All patients remained on optimal pharmacological treatment as summarized in Table 2.

The presence, severity, and changes in oxygen stress during CRT were assessed by TBARS, and expressed as MDA concentration both in plasma and in red blood cells (Buege-Aust method [21]). The Buege-Aust method was published in 1978 and up to date it has been a widespread test for detection of products of lipid peroxidation. The method is based on estimation of aldehydic products of lipid peroxidation and their ability to react with thiobarbituric acid. This products - "thiobarbituric acid reactive substances" (TBARS) — can be easily detected by spectrophotometry [22].

Changes in antioxidant activity were assessed by measuring the activity of CAT, SOD and GPx in red blood cells. The above-mentioned enzymatic activities were determined according to Beers-Sizer [23], Misra-Fridovich [24] and Paglia-Valentine [25] methods, respectively.

The concentration and activity of the above-mentioned substances were analyzed directly prior to CRT implantation (in the morning on the day of the procedure), and then $2-5$ days after the procedure (acute phase) and after 6 months. Fasting blood samples were acquired from the antecubital vein.

Follow-up was performed after 6 months and included clinical (anamnesis, physical examination, electrocardiogram), echocardiographic and implanted device assessment. Clinical improvement was defined as NYHA class reduction and resolving of HF physical symptoms and signs such as edema, pulmonary congestion evidenced by pulmonary crackles, and S3 gallop.

\section{Statistical analysis}

Statistical analysis was performed using the Statistica, version 10.0 package (StatSoft, Tulsa,

Table 2. Pharmacological treatment.

\begin{tabular}{lccc}
\hline Classes of drugs & $\begin{array}{c}\text { Before CRT } \\
(\mathbf{n}=\mathbf{5 1 )}\end{array}$ & $\begin{array}{c}\text { 2-5 days after CRT } \\
(\mathbf{n}=\mathbf{4 9})\end{array}$ & $\begin{array}{c}\mathbf{6} \text { months after CRT } \\
\text { (n }=44)\end{array}$ \\
\hline Beta-blockers & $48(94.1 \%)$ & $49(96 \%)$ & $44(100 \%)$ \\
Angiotensin converting enzyme & $39(76.4 \%)$ & $41(80.3 \%)$ & $33(75 \%)$ \\
Angiotensin receptor blocker & $9(17.6 \%)$ & $9(17.6 \%)$ & $8(18.1 \%)$ \\
Diuretics & $44(86.2 \%)$ & $49(96 \%)$ & $41(93.1 \%)$ \\
Aldosterone antagonists & $41(80.3 \%)$ & $46(90.1 \%)$ & $39(88.6 \%)$ \\
Digoxin & $7(13.7 \%)$ & $6(11.7 \%)$ & $7(15.9 \%)$ \\
Nitrates & $6(11.7 \%)$ & $5(9.8 \%)$ & $5(11.3 \%)$ \\
Acetylsalicylic acid & $36(70.5 \%)$ & $43(84.3 \%)$ & $36(81.8 \%)$ \\
Statins & $38(74.5 \%)$ & $44(86.2 \%)$ & $40(90.9 \%)$ \\
Amiodarone & $9(17.6 \%)$ & $7(13.7 \%)$ & $5(11.3 \%)$ \\
Vitamin K antagonists & $1(1.9 \%)$ & $6(11.7 \%)$ & $5(11.3 \%)$ \\
Clopidogrel & $8(15.6 \%)$ & $10(19.6 \%)$ & $4(9.0 \%)$ \\
Low-molecular-weight heparin & $11(21.5 \%)$ & & \\
\hline
\end{tabular}

CRT — cardiac resynchronization therapy 


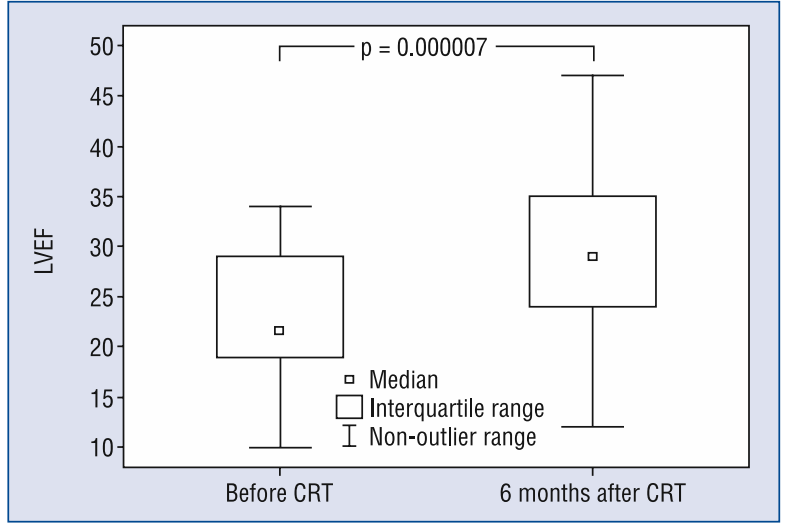

Figure 1. Changes in left ventricular ejection fraction (LVEF) before cardiac resynchronization therapy (CRT) implantation and after 6 months of pacing.

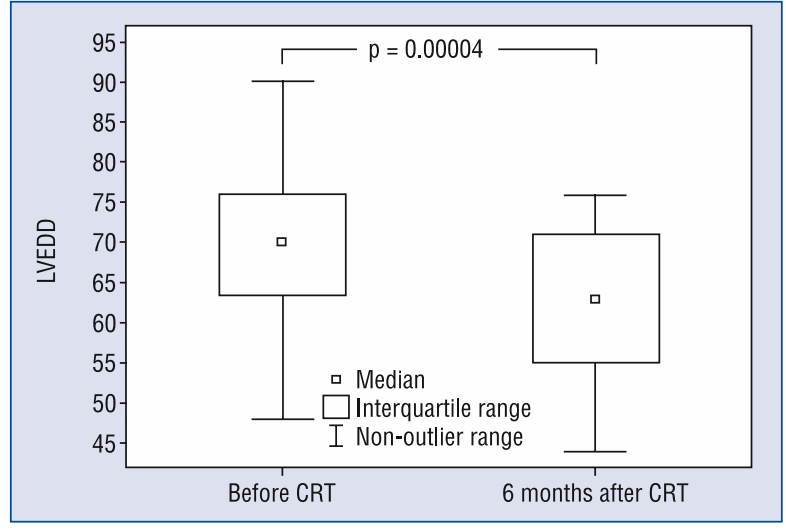

Figure 2. Changes in left ventricular end diastolic diameter before cardiac resynchronization therapy (CRT) implantation and after 6 months of pacing.

Table 3. Thiobarbituric acid-reactive substances plasma concentration and catalase, superoxide dismutase, and glutathione peroxidase activity in red blood cells.

\begin{tabular}{lccc}
\hline & Before CRT & 2-5 days after CRT & 6 months after CRT \\
\hline pMDA [nmolMDA/mL] & $0.41 \pm 0.1$ & $0.48 \pm 0.12 \# \boldsymbol{\Delta}$ & $0.39 \pm 0.05 * \boldsymbol{\Delta}$ \\
rMDA [nmolMDA/gHb] & $30.97 \pm 9.6$ & $30.84 \pm 8.9$ & $28.49 \pm 14.2$ \\
Catalase [U/gHb] & $68.06 \pm 7.5$ & $71.49 \pm 13.1$ & $63.09 \pm 11.9 * \boldsymbol{\Delta}$ \\
Superoxide dismutase $[\mathrm{U} / \mathrm{gHb}]$ & $1013.32 \pm 149.7$ & $933.07 \pm 114.5$ & $848.13 \pm 223.2 * \boldsymbol{\Delta}$ \\
Glutathione peroxidase $[\mathrm{U} / \mathrm{gHb}]$ & $9.16 \pm 5.5$ & $9.26 \pm 4.9$ & $5.87 \pm 3.3 * \boldsymbol{\Delta}$ \\
\hline
\end{tabular}

*Statistical significance $(p<0.05)$ between measurements prior to and 6 months after CRT; \#Statistical significance $(p<0.05)$ between measurements prior to and 2-5 days after CRT; $\boldsymbol{\Delta}$ Statistical significance $(p<0.05)$ between measurements $2-5$ days after and 6 months after $\mathrm{CRT}$; CRT - cardiac resynchronization therapy; pMDA — plasma concentration of malondialdehyde; rMDA — red blood cells concentration of malondialdehyde

OK, USA). The Shapiro-Wilk test demonstrated non-normal distribution of the investigated variables. Therefore, continuous variables were reported as median values and interquartile ranges. The Wilcoxon signed-rank test was used to compare continuous variables at different time points. Values of $p<0.05$ were considered statistically significant.

\section{Results}

Cardiac resynchronization therapy pacemakers (CRT-P) were implanted in $9(17 \%)$ patients, while $42(82 \%)$ patients received cardiac resynchronization therapy defibrillators (CRT-D). During the entire time of the follow-up 7 (14\%) patients died 5 of them due to cardiovascular events. The median percentage of biventricular pacing assessed by the data from implanted devices was $99.29 \%$ (ranging 99.15-100\%) at the end of 6-month follow-up.

Prior to CRT implantation $40(95 \%)$ patients were in NYHA class III and $2(5 \%)$ in NYHA class IV.
After 6 months 37 (88\%) patients improved to NYHA class II, while $5(12 \%)$ remained in the same NYHA class as before the implantation (non-responders).

Echocardiographic data are summarized in Figures 1 and 2. LVEF increased from median $21.5 \%$ (range 18.5-27.75) before the CRT device implantation to 29\% (range 24-35) at 6-month follow-up $(\mathrm{p}<0.05)$. Respectively, left ventricular end-diastolic diameter (LVEDD) significantly decreased from a median of $69.5 \mathrm{~mm}$ (range 63-75.75) to $63 \mathrm{~mm}$ (range 55.5-70) $(\mathrm{p}<0.05)$.

MDA concentrations in plasma and the red blood cells, as well as the activity levels of CAT, SOD and GPx prior to CRT, after $2-5$ days and after 6 months are presented in Table 3 and Figures 3-7.

During the acute period of the study (2-5 days after the implantation) a significant increase in MDA plasmatic concentration was observed $(\mathrm{p}<0.02)$. Similarly, in the acute period, activities of the remaining enzymes, except for SOD, showed a trend towards increase, but the changes were statistically insignificant ( $p>0.05)$. 


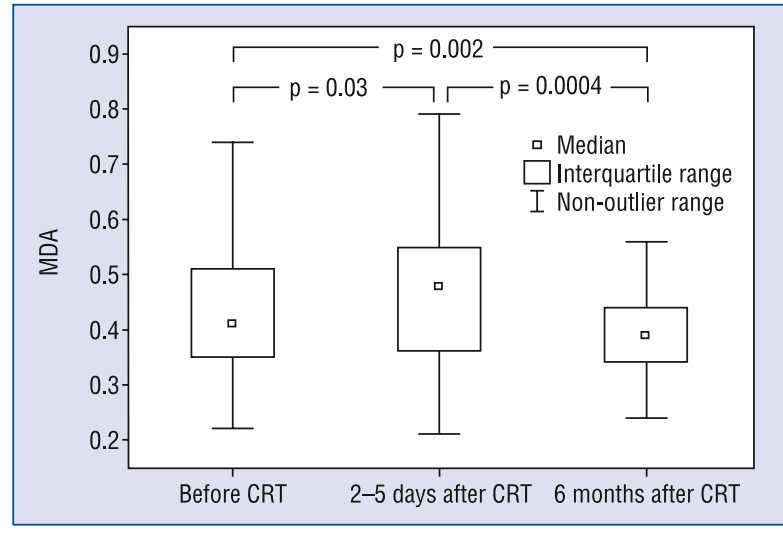

Figure 3. Malondialdehyde (MDA) plasma concentration before and after cardiac resynchronization therapy (CRT) implantation.

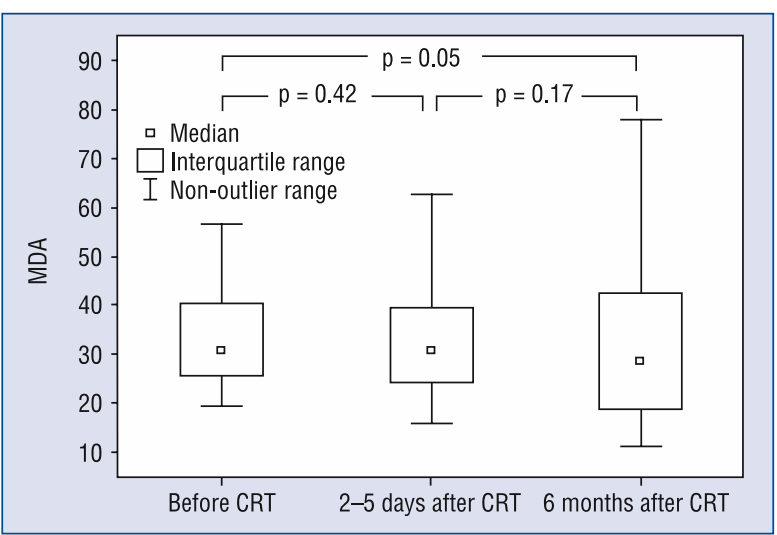

Figure 4. Malondialdehyde (MDA) red blood cells concentration before and after cardiac resynchronization therapy (CRT) implantation.

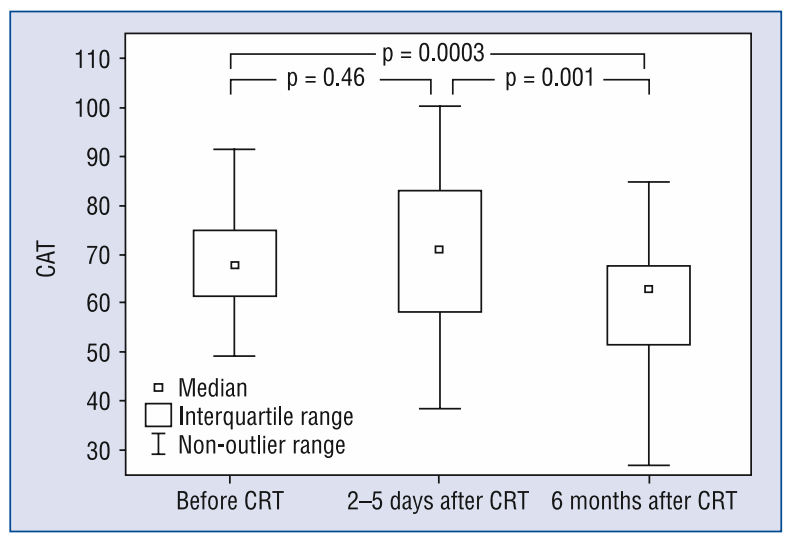

Figure 5. Catalase (CAT) activity in red blood cells before and after cardiac resynchronization therapy (CRT) implantation.

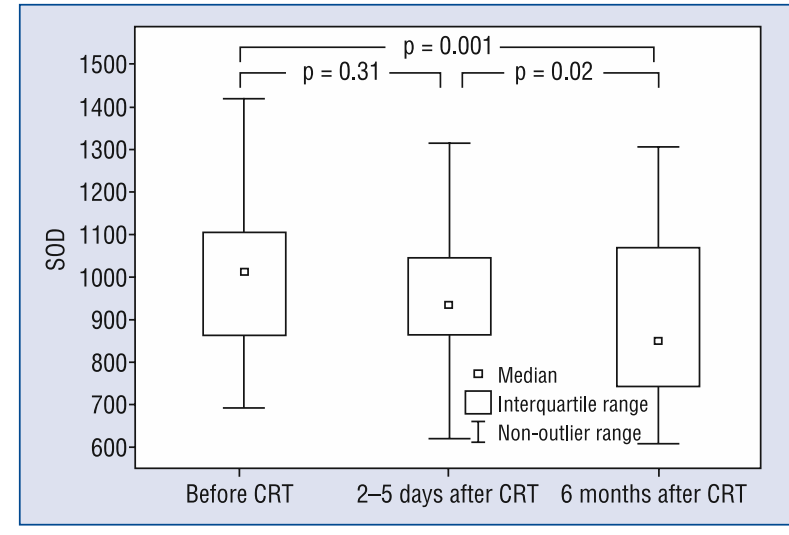

Figure 6. Superoxide dismutase (SOD) activity in red blood cells before and after cardiac resynchronization therapy (CRT) implantation.

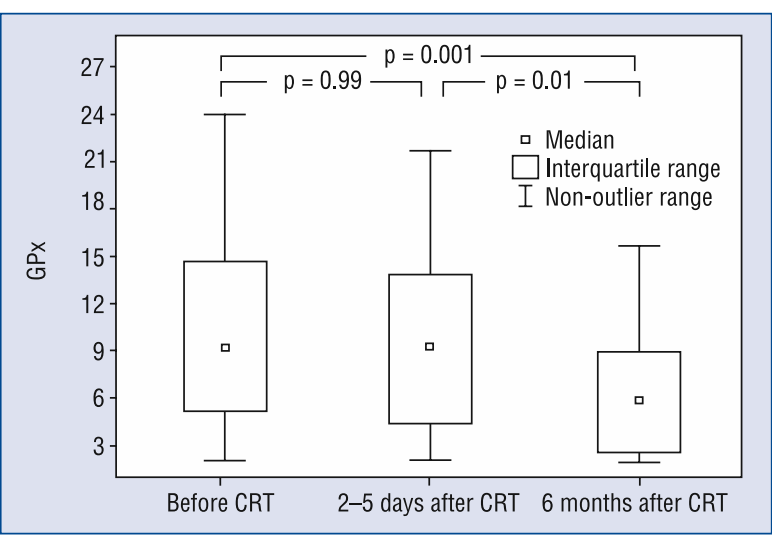

Figure 7. Glutathione peroxidase (GPx) activity in red blood cells before and after cardiac resynchronization therapy (CRT) implantation.

After 6 months of CRT pacing MDA plasma concentration and the activity levels of each antioxidant enzyme measured in red blood cells (CAT, SOD, GPx) showed a statistically significant reduction $(\mathrm{p}<0.05)$. Analogous changes concerning MDA concentrations in red blood cells remained statistically insignificant.

\section{Discussion}

Heart failure per se, regardless of its etiology, is associated with elevation of oxidative stress markers (i.e. increase in TBARS concentration, indicative of ROS presence). There is often a directly proportional correlation between the magnitude of this elevation and the degree of HF progression [16, 20, 26-31]. 
Polidori et al. [28] reported higher plasma concentrations of MDA in NYHA class III patients as compared with NYHA class II patients, as well as an inverse correlation between MDA concentration and LVEF. Similar findings were presented by Belch et al. [26] and Keith et al. [30]. Additionally, a decrease in MDA concentration is a common finding coexisting with clinical improvement in this group of patients.

In a study by Castro et al. [32], a decline in MDA plasma concentration in patients with clinical improvement occurred after medical therapy, in contrast to MDA concentration in non-responders.

In our study group, improvement in the clinical status of patients and reverse remodeling after 6 months of CRT pacing were associated with attenuation of oxidative stress as assessed by MDA plasma concentration.

As described in literature, CRT results in reverse remodeling of the $\mathrm{LV}$ and an increase in $\mathrm{LVEF}$ by $6-11 \%$ during the first $3-6$ months [33-37]. According to our observations, resynchronization therapy leads to reduction of oxidative stress in the failing heart as expressed by decreasing concentrations of lipid peroxidation products.

Oxidative stress in the failing heart results from imbalance between excess of free radicals production and coexisting impairment of antioxidant defenses in myocardium. Antioxidant activity may be assessed by measuring activities of specific enzymes (the most important of which are intracellular enzymes such as CAT, SOD, or GPx) or non-enzymatic substances such as vitamins $\mathrm{C}$ or $\mathrm{E}$. Since literature data regarding changes of antioxidant substances in chronic HF mainly concern antioxidant enzymes, in contrast to non-enzymatic mechanisms [33], we decided to research into the former.

Depressed activity of the antioxidant enzymes usually occurs in response to elevation of ROS concentrations in HF [21]. The majority of authors explain it by enhanced participation of the antioxidant system in neutralization of ROS. Radovanovic et al. [38] stated that antioxidant enzymes activity can also change in response of progression of HF. Moreover, there can be different tendencies regarding different enzymes. GPx significantly decreases along with progression of NYHA class and LVEDD, as well as with lowering of LVEF. SOD, in turn, demonstrates opposite direction of changes, with a significant increase in its activity along with progression of HF [38].

Interestingly, in our study, the activity of antioxidant enzymes responsible for ROS neutralization after 6 months of CRT therapy decreased when compared with baseline values. It seems that this decrease may be associated with the reduction of oxygen stress during the therapy. However, our data contradict observations of many researchers who reported depressed antioxidant activity as a marker of elevation of reactive oxygen specific for $\mathrm{HF}$ and increasing antioxidant enzymes activity in response to treatment [26]. It is interesting to note that our results concern the activity of enzymes measured inside the cells (intracellular antioxidant activity), but not in plasma.

\section{Limitations of the study}

It should also be underlined that the limited duration of follow-up in our study does not allow to determine whether further clinical improvement of the patients will be correlated with further changes in MDA concentrations or an increase in the activity of the antioxidant enzymes.

\section{Conclusions}

Plasma activities of antioxidant enzymes are probably related to a systemic reaction to oxidative stress, but the intra-cellular decrease in the above-mentioned enzymes may suggest that there is no need for antioxidant activity enhancement due to reduction of oxygen stress at cellular level.

\section{Conflict of interest: None declared}

\section{References}

1. Dickstein K, Vardas PE, Auricchia A et al. 2010 Focused Update of ESC Guidelines on device therapy in heart failure An update of the 2008 ESC Guidelines for the diagnosis and treatment of acute and chronic heart failure and the 2007 ESC guidelines for cardiac and resynchronization therapy Developed with the special contribution of the Heart Failure. Association and the European Heart Rhythm Association. Eur Heart J, 2010; 31: 2677-2687.

2. Machado M, Falcao LM, Ravara L. Sudden death prevention in heart failure. Rev Port Cardiol, 2006; 25:727-762.

3. Moss AJ. Saving lives and reducing inappropriate device therapy: The MADIT family of implantable cadioverter-defibrillator and cardiac resynchronization therapy trials. Cardiol J, 2013; 20: 217-219.

4. Jeevanantham V, Daubert JP, Zareba W. Cardiac resynchronization therapy in heart failure patients: An update. Cardiol J, 2009; 16: 197-209.

5. Bristow MR, Saxon LA, Boehmer J et al. Cardiac-resynchronization therapy with or without an implantable defibtillator in advanced chronic heart failure. N Engl J Med, 2004; 350: 2140-2150.

6. Cleland JG, Daubert JC, Erdmann E et al. The effect of cardiac resynchronization on morbidity and mortality in heart failure. N Engl J Med., 2005; 352: 1539-1549. 
7. Linde C, Abraham WT, Gold MR, Sutton MSJ, Ghio S, Daubert C. Randomized trial of cardiace resynchronization in mildly symptomatic heart failure patients and in asymptomatic patients with left ventricular dysfunction and previous heart failure symptoms. J Am Coll Cardiol, 2008; 52: 1834-1843.

8. Higgins SL, Hummel JD, Niazi IK et al. Cardiac resynchronization therapy for the treatment of heart failure in patients with intraventricular conduction delay and malignant ventricular tachyarrhythmias. J Am Coll Cardiol, 2003; 42: 1454-1459.

9. Sutton MG, Plappert T, Abraham WT et al. Effect of cardiac resynchronization therapy on left ventriculat size and function in chronic heart failure. Circulation, 2003; 107: 1985-1990.

10. Cazeau S, Leclercq C, Lavergne $\mathrm{T}$ et al. Effects of multisite biventricular pacing in patients with heart failure and intraventricular conduction delay. N Engl J Med, 2001; 344: 873-880.

11. Schutte F, Ludorff G, Grove R, Kraing W, Thale J. Atrioventricular node ablation is a not prerequisite for cardiac resynchronization therapy in patients with chronic atrial fibrillation. Cardiol J, 2009; 16: $246-249$.

12. Fazelifar AF, Bonakdar HF, Alizadeh K et al. Relationship between QRS complex notch and ventricular dyssynchrony in patients with heart failure and prolonged QRS duration. Cardiol J, 2008; 15: 351-356.

13. Jeevanantham V, Zareba W., Navaneethan S et al. Metaanalysis on effects of cardiac resynchronization therapy in heart failure patients with narrow QRS complex. Cardiol J, 2008; 15: 230-236.

14. Moss AJ, Hall WJ, Cannom DS et al. Cardiac-resynchronization therapy for the prevention of heart failure events. N Engl J Med., 2009; 361: 1329-1338.

15. Tarquini R, Guerra CT, Porciani MC et al. Effects of cardiac resynchronization therapoy on systemic inflamation and neurohormonal pathways in heart failure. Cardiol J, 2009; 16: 545-552.

16. Pail ML. The NO/ONOO-cycle as the central cause of heart failure. Int J Mol Sci, 2013; 14: 22274-22330.

17. Singal PK, Khaper N, Palace V, Kumar D. The role of oxidative stress in the genesis of heart failure. Cardiovasc Res, 1998; 40: 426-432.

18. Grieve DJ, Shah AM. Oxidative stress in heart failure. More than just damage. Eur Heart J, 2003; 24: 2161-2163.

19. Tsutsui H. Oxidative stress in heart failure: The role of mitochondria. Internal Med, 2001; 40: 1177-1182.

20. MMc Murray J, Chopra M, Abdullah I, Smith WE, Dargie HJ. Evidence of oxidative stress in chronic heart failure in humans. Eur Heart J, 1993; 14: 1493-1498.

21. Buege JA, Aust SD. Microsomal lipid peroxidation. In: Fleisher S, Packer I eds. Methods in enzymology. Academic Press, New York 1978: 52, 302-310.

22. Devasagayam TPA, Boloor KK, Ramasarma T. Methods for estimating lipid peroxidation: An analysis of merits and demerits. Indian J Biochem Biophys, 2003; 40: 300-308.

23. Beers RF, Sizer IW. A spectrophotometric method for measuring the breakdown of hydrogen peroxide by catalase. J Biol Chem, 1952; 195: 133-140.
24. Misra HP, Fridovich I. The role of superoxide anion in the autooxidation of epinephrine and a simple assay for superoxide dismutase. J Biol Chem, 1972; 247: 3170-3175.

25. Paglia DE, Valentine WN. Studies on the quantitative and qualitative characterization of erythrocyte glutathione peroxidase. J Lab Clin Med, 1967; 70: 158-169.

26. Belch JJF, Bridges AB, Scott N, Chopra M. Oxygen free radicals and congestive heart failure. Br Heart J, 1991; 65: 245-248.

27. Perez O, Castro P, Diaz-Araya G et al. Persistence of oxidative stress after heart transplantation: A comparative study of patients with heart transplant versus chronic stable heart failure. Rev Esp Cardiol, 2002; 55: 831-837.

28. Polidori MC, Savino K, Alunni G et al. Plasma lipophilic antioxidants and malondialdehyde in congestive heart failure patients: relationship to disease severity. Free Radic Biol Med, 2002; 32: 148-152.

29. Diaz-Velez CR, Garcia-Castiňeiras S, Mendoza-Ramos E, Hernandez-Lopez E. Increased malondialdehyde in peripheral blood in patients with congestive heart failure. Am Heart J, 1996; 131: 146-152.

30. Keith M, Geranmayegan A, Sole MJ et al. Increased oxidative stress in patients with congestive heart failure. J Am Coll Cardiol, 1998; 31: 1352-1356.

31. Campolo J, De Maria R, Caruso R et al. Blood glutathione as independent marker of lipid peroxidation in heart failure. Int J Cardiol, 2007; 117: 45-50

32. Castro PF, Diaz-Araya G, Nettle D et al. Effects of early decrease in oxidative stress after medical therapy in patients with class IV congestive heart failure. Am J Cardiol, 2002; 89: 236-239.

33. St John Sutton M, Plappert T, Abraham WT et al. Effect of cardiac resynchronization therapy on left ventricular size and function in chronic heart failure. Circulation, 2003; 107: 1985-1990.

34. Bax JJ, Bleeker GB, Marwick TH et al. Left ventricular dyssynchrony predicts response and prognosis after cardiac resynchronization therapy. J Am Coll Cardiol, 2004; 44: 1834-1840.

35. Yu CM, Bleeker GB, Fung JWH et al. Left ventricular reverse remodeling but not clinical improvement predicts long-term survival after cardiac resynchronization therapy. Circulation, 2005; 112: 1580-1586.

36. Bleeker GB, Schalij MJ, Holman ER, Steendijk P, van der Wall EE, Bax JJ. Cardiac resynchronization therapy with systolic left ventricular dysfunction and symptoms of mild heart failure secondary to ischemic or nonischemic cardiomyopathy. Am J Cardiol, 2006; 98: 230-235.

37. Zhang Q, Fung JWH, Auricchio A et al. Differential change in left ventricular mass and regional wall thickness after cardiac resynchronization therapy for heart failure. Eur Heart J, 2006; 27: 1423-1430.

38. Radovanovic S, Savic-Radojevic A, Pljesa-Ercegovac M et al. Markers of oxidative damage and antioxidant enzyme activities as predictors of morbidity and mortality in patients with chronic heart failure. J Cardiac Fail, 2012; 18: 493-501. 